TRAUMATIC BRAIN INJURY

\section{TBI could have long-term effects on sleep-wake cycle}

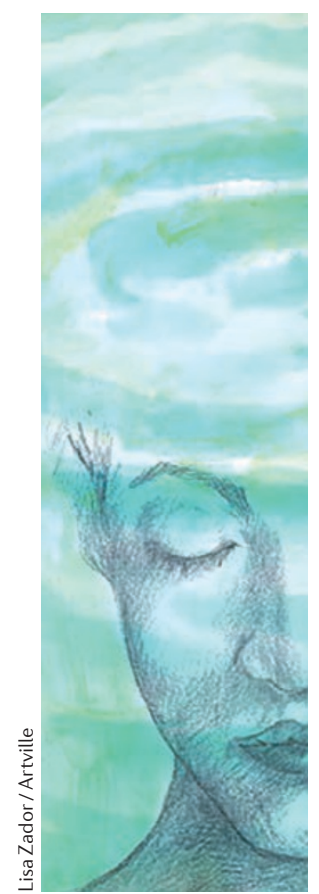

Traumatic brain injury (TBI)

disrupts the sleep-wake cycle and increases daytime sleepiness for at least 1.5 years after the initial injury, according to a recently published follow-up study. The findings suggest that TBI survivors might be at increased risk of accidents and have impaired quality of life long after the injury.

Several studies have reported that TBI survivors experience a greater need for sleep and increased daytime sleepiness; however, the majority of those studies have been restricted to the acute phase of TBI recovery and relied on self reports. In the new study, Lukas Imbach and colleagues used actigraphy and polysomnography to obtain objective measures of sleep duration and quality, and sleep latency tests to assess chronic excessive daytime sleepiness.
18 months after injury, sleep need was higher (mean sleep duration $8.1 \mathrm{~h}$ ) in 31 participants with previous TBI than in 42 healthy controls (mean sleep duration $7.1 \mathrm{~h}$ ). Excessive daytime sleepiness was strikingly more common (67\%) in the TBI group than in controls (19\%).

Interestingly, participants with a TBI did not self-report sleep-wake alterations. "Patients significantly underestimated excessive daytime sleepiness and sleep need," Imbach comments. The finding implies that subjective estimates of sleep need and sleepiness are unreliable in patients with TBI. Moreover, individuals recovering from TBI might be at increased risk of traffic and occupational accidents long after the injury.

The study by Imbach and co-workers was not designed to unravel the mechanisms of TBI-associated sleep-wake cycle alterations. According to Imbach, different mechanisms could be involved in sleep-wake perturbations in the acute and chronic phase after TBI.
Previous studies have suggested that sleep has an important role in promoting the clearance of amyloid- $\beta(A \beta)$ and other harmful substances from the brain. Although the role of sleep in $\mathrm{A} \beta$ clearance has not been studied specifically in the context of TBI in humans, Imbach suggests that increased sleep need in the acute phase after TBI might be linked to neuronal recovery.

However, the reason for a persistent increase in sleep need after TBI is not clear. "We hypothesize that TBI could also induce permanent damage to highly vulnerable sleep-relevant cerebral centres," Imbach contends. Such damage could result in long-lasting sleep-wake disorders.

Hemi Malkki

ORIGINAL ARTICLE Imbach, L. L. et al. Sleepwake disorders persist 18 months after traumatic brain injury but remain underrecognized. Neurology http://dx.doi.org/10.1212/ WNL.0000000000002716 (2016) FURTHER READING Edlow, B. L. \& Lammers, G. J. Bringing sleep-wake disorders out of the dark.

Neurology http://dx.doi.org/10.1212/

WNL.0000000000002710 (2016) 